\title{
Infusing Meaning into Social Wearables: Lessons from Sentimental Jewelry
}

\author{
Yulia Silina \\ Queen Mary University of London \\ Mile End Rd, London E1 4NS, UK \\ CuriousYulia@gmail.com
}

\author{
Anne Hsu \\ Queen Mary University of London \\ Mile End Rd, London E1 4NS, UK \\ Anne.Hsu@qmul.ac.uk
}

\begin{abstract}
Wearable devices play an increasingly important role in maintaining and improving the sense of connectedness between loved-ones. However, despite affording numerous novel interactions and possibilities, wearables are easily discarded. And their aesthetic and functional designs are often gadget-like, or rely on cultural clichés, underutilizing the rich history of their "analogue" counterpart of sentimental jewelry. In this paper, we conduct a content analysis study to understand practices associated with the use of sentimental jewelry. We uncover that in contrast to social wearables, analogue artefacts are rarely paired and are intended to be worn by female partners, friends and family members. They are seldom personalized, and either explicitly convey affection, good luck or motivation or allow users to infuse their own meaning into them, or. These findings give us insights that could increase relevance of social wearables for their users, and as a result, enhance user's wellbeing and connectedness to their loved-ones.
\end{abstract}

Wearables, gadgets, wellbeing, digital jewellery, computational jewelry, computer mediated communication.

\section{INTRODUCTION}

\subsection{Mediating Intimate Relationships through Technology}

The field of Computer-Mediated Communication (CMC) encompasses multitude of computational artefacts that allow people to communicate with one-another. Our paper concerns itself with a specific subset in this area of research that focuses on mediating intimate relationships through technology (detailed overview in Hassenzahl et al., 2012). The artefacts and associated systems in this field are characterized by being explicitly dedicated to communication between loved-ones. They are often of non-verbal and sentimental nature, and predominantly focus on romantic partners in longdistance relationships (LDR). Among many and varied artefacts that mediate intimate relationships, some take form of jewelry-like social wearables (detailed overview in Silina \& Haddadi, 2015).

\subsection{Multidisciplinary Clarifications}

The fields of CMC and social wearables are highlymultidisciplinary. Thus, before delving into the practices, associated with sentimental jewelry in this context, it is important to establish a common ground. (a) The term Jewelry "refers to forms of personal adornment, worn on the body". (b) Jewelry-like wearables refers to "a subset of wearables that occupy traditional places on the body as jewelry, but do not necessarily look like jewelry" (Silina \& Haddadi, 2015). (c) Jewelry-like wearables that actually look like jewelry belong to the field of Fashion Tech (Seymour, 2008; Silina \& Haddadi, 2015) and are often called smart jewelry (a.k. computational jewelry (Silina \& Haddadi, 2015), or digital (Wallace, 2007) and interactive (Versteeg et al., 2016) jewellery). (d) Social wearables are wearable devices that mediate relationships and belong to the field of CMC. Here we use social wearables as a shorthand for jewelry-like devices that mediate relationships between loved-ones. (e) Sentimental jewelry is a form of mementos, keepsakes and love-tokens that look like jewelry)

As other sentimental artefacts, sentimental jewelry broadly falls into three categories: (1) Artefacts intentionally acquired by wearier. (2) Artefacts that land in wearer's possession unintentionally. (3) Artefacts that were intentionally given to / or shared with the wearer by another person. As this paper concerns with mediation of intimate relationships, we focus on the last category. In this context, paired artefacts are intended to be worn by both parties and afford 2-way imaginary or technological "communication" (for example friendship bracelets or connected devices). Singular artefacts (for example lockets) are intended for a single wearer and afford 1-way "communication". 


\subsection{Social Wearables as Mediators of Relationships}

According to Silina \& Haddadi, there are distinct differences between form and function of social wearables, created in academic as opposed to commercial environments. But, overall, both users and potential use scenarios of social wearables are still poorly understood by both communities. Most social wearables that originated in academic research have a gadget-like form factor and were developed as paired devises, intended to be worn by men and women alike. Many of these are bulky and not usable outside the lab. Although there are some smart jewelry artefacts, many of these are designed for specific individuals in specific user studies, giving rich incites but limiting replicability. Although there are few notable exceptions that explore mother-daughter relationship (Wallace, 2007) and communications among the networks of children (Kikin-Gil, 2005; Kettley, 2007), most works reflect the approach of the broader field of tech mediated relationships and focus on communication between romantic partners in LDR. In sharp contrast, commercial social wearables are mass produced for everyday use and designed to mediate a wide variety of interpersonal relationships. However, they are increasingly designed as smart jewelry and aimed at women as wearers. Their systems frequently comprised of the smart phone app with some customization, and a singular wearable device that receives basic form of VIP notifications through vibration and/ or light, like in ringly.com and plumora.com.

\subsection{Historical Context of Sentimental Jewelry}

It is common to use historical record of sentimental artefacts as a shorthand cliché in the design of systems for mediation of intimate relationships through technology. But in the context of social wearables, most of the Western-centred literature on sentimental jewelry focuses on priceless bespoke artefacts that were lucky to survive (Scarisbrick \& Fenton, 2013), and the records stop with an advent of mass production in early $20^{\text {th }}$ century. This creates a number of concerns about the validity of use of such a shorthand in design of computational artefacts.

In historical terms, most survived sentimental jewelry dates from as far back as Ancient Greece up to $19^{\text {th }}$ century. Majority have either inscriptions or portraits and employ recognizable archetypal symbols such as hearts and wings that originated in the early cult of Aphrodite and percolate to modern days (Munn, 1993). Throughout the times, these artefacts have been used for expression of love and commitment as well as for luck and protection by emotionally involved people, regardless of their gender and relationship type (Bury, 1986). Specific traditions and exact purposes of these artefacts varied over different époques with some of the customs, enduring and others disappearing with changing fashions (Tait \& Andrews, 2007).

Considering the growing use and benefits of social wearables in maintaining relationships between loved-ones, there is a need to address the disposable nature and low adoption of gadget-like wearables. The enduring practice of exchanging sentimental artefacts to maintain the sense of connectedness between loved-ones can offer us invaluable lessons that could be applied to creating meaningful long lasting "digital" artefacts. There is a revived interest in the modern use of sentimental jewelry (Ahde-Deal, 2013; Rana, ongoing) that illustrate current values and stories that wearers of all genders, backgrounds and ages infuse into their sentimental jewelry. And our paper calls to consider the lessons that sentimental jewelry can offer in design of meaningful social wearables.

\section{RESEARCH AIM}

Although there are many parallels between the use of "analogue" sentimental jewelry and social wearables that are worth investigating, our current paper aims to examine the types of modern users and use scenarios of sentimental jewelry though a set of following exploratory questions: (Q1) What categories of users might currently exist other than romantic couples? (Q2) Is sentimental jewelry intended to be worn by men as much as by women? (Q3) Are current sentimental jewelry artefacts paired, or intended to be worn only by one person? (Q4) What use scenarios of sentimental jewelry might currently exist other than expression of affection? (Q5) How are these use scenarios expressed on the jewelry? (Q6) Are current sentimental jewelry artefacts as bespoke or customized as they were in the past?

\section{STUDY}

\subsection{Method}

To explore our research aim and to get a broader up-to-date understanding of sentimental jewelry use, we conducted a study that looked at the consumer market of artefacts, distributed through two major online retailers: Etsy.co.uk (that stocks bespoke and vintage jewelry) and Amazon.co.uk (that stocks mass-produced jewelry). Both websites were UK based, and were searched from Google Chrome in "incognito" mode. To reduce the potential bias and to align with popularity of wristworn wearables (Seymour, 2008), we choose to focus on bracelets. Sample was taken based on the term "bracelet" form consecutive results, automatically displayed "by relevance" on Etsy and 
in "featured" section on Amazon. We did not discriminate based on materials and monetary value of the artefacts. Although the automated search and analysis of images and meta-data was considered, the range of artefacts and variety of photography styles did not allow that. Thus, the content of images and tags was manually evaluated by researchers. A jewelry item was considered sentimental if at least one of following characteristics was present: (1) Item's description indicated that it was a keepsake or gift for a specific person or occasion. (2) If inscription on artefact indicated that it was for someone. (3) If the artefact contained traditionally used archetypal symbols (hearts, love birds, etc.).

Once identified, each artefact was categorized by the type of intended recipient (wearer), recipient's gender, "communication" practices (paired, single, etc.) and reasons for intended use. For example: bracelet with a heart and inscription "best sister", was places under sub-categories sister; female; 1 way (single); inscription, heart. List of categories and sub-categories was expanded as new categories emerged in examined artefacts. Artefacts were called generic if they contained archetypal sentimental symbols without stating specific use.

\subsection{Results}

Overall, we examined a sample of 4,492 pieces (10\% of search results on Etsy and $1 \%$ on Amazon) and identified about 16\% (698) of them that had characteristics of sentimental jewelry.

Q1. Categories of intended wearers: Overall 740 instances of wearer categories emerged with Some pieces being intended to more than one type of wearier. Out of these, about a third (265) of sentimental artefacts were generic. The rest fell into three categories of intended wearers: romantic partners (211), friends (115) and family (149). The family category of wearers further broke down into distinct categories, with mother (50), daughter (35) and sisters (26) being most prevalent (Figure 1).

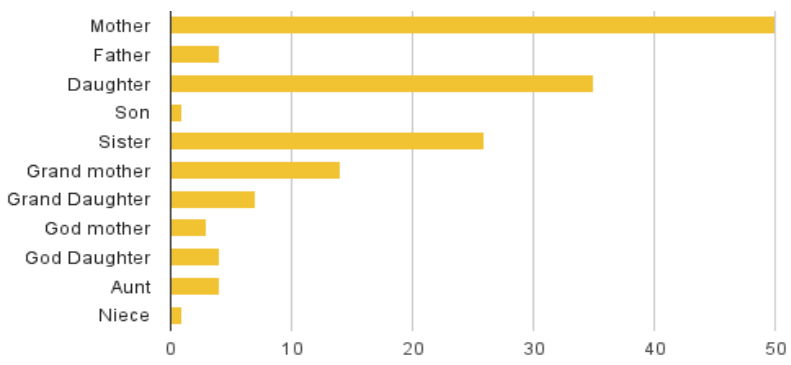

Figure 1: Intended recipients in family group

Q2. Gender of wearers: Almost 95\% (661) of all identified sentimental jewelry was intended to be worn by women. The small remainder was designed to be worn by men (13) or to be unisex (24).
Q3. Communication practices: Only 21 artefacts were paired, with near 97\% (677) singular artefacts, intended to be given to and worn by one person.

Q4. Intended use: About 38\% (265) were designed to be generic. Almost a half of artefacts (327) expressed affection. Three other categories emerged: long distance commitment (8), motivation (59) and wishes of good luck (39).

Q5. Manifestation of intended use: The intended use was conveyed through either archetypal symbols, inscriptions or some combination of these. Examined artefacts contained 499 symbols. Most of these (460) were love and commitment symbols (eternity, love birds, love knot, etc), with heart being most prevalent (271). Remaining 39 symbols associated with good luck (clover, nazar's eye, etc). Among identified 419 inscriptions, almost a half of them (203) were sentimental quotes, about a third (157) personalization, and finally (59) motivational.

Q6. Personalization: Looking at artefacts themselves, only about $22 \%$ (155) of artefacts were personalized, majority relying on recognizable archetypal symbols or off-the-shelf inscriptions, like 'best mom', 'love you to the moon and back' etc.

\section{DISCUSSION \& FUTURE WORK}

\subsection{Form Factor \& Multidisciplinarity}

It is hardly possible to capture the entire range of sentimental artefacts, as people commonly exchange gifts with or without archetypal symbols. However, even with this limitation, it is evident that in the modern Western-centered cultural context, sentimental jewelry is still used to maintain presence of loved-ones, with a large portion of commercial and bespoke jewelry market dedicated to it. Unlike gadgets that are easily disposable, people keep jewelry even when it does not have financial value. This is particularly true for sentimental jewelry (Ahde-Deal, 2013). This suggests that jewelry form-factor might be a better alternative to gadgets, prevalent in current academic and commercial social wearables.

Although, fashions for types and styles of jewelry are transient in their nature, this could be accounted for by the collaboration of research teams with jewelry designers (Seymour, 2008; Silina \& Haddadi, 2015). Jewelry designers do not only understand context and aesthetic preferences of different user-groups, but they ca also resolve ergonomic and production issues that often make academic prototypes of wearables gimmicky and impractical for every-day use.

With a multitude of wrist-worn devices that come to the market, the wrist is a valuable real-estate (Kim et al., 2016). Thus, designers of social wearables may 
want to consider other types of jewelry, such as rings, broaches, earrings and necklaces. Admittedly, these require greater miniaturization of technology, and although hardware prototyping platforms are getting smaller, this is still a limiting factor for researchers. However, in the artefacts like ouraring.com and earosmart.com, industry have resolved many of the challenges of wearable computing, outlined Thad Starner over a decade ago (Starner, 2001). Industry also have accesses to a big pull of users, but unfortunately the interaction explorations within industry are stifled by market factors and practicalities. These suggests a rich ground for mutually-beneficial collaboration between $\mathrm{HCl}$ research community and manufacturers of social wearables.

\subsection{Personalization \& Flexibility}

It is interesting that unlike earlier sentimental jewelry, modern artefacts are seldom personalized, echoing commercial artefacts and contrasting with bespoke or stationary artefacts in academic research. Designing social wearables without the need to be unique for each use-case opens possibilities of testing them outside of laboratory in multiple user-pairs and groups, which was difficult to do until now. The study results also indicate that modern sentimental jewelry is rarely paired. This aligns with commercial wearables that frequently intended for are a single jewelry-like device to communicate with other platforms, like mobile phones of loved-ones. And again, these findings contrast with academic research, where social wearables are often intended to be worn by both users. In this context, it would be interesting to explore paired social wearables like HB Ring by thetouchx.com. As well self-contained social wearables, like CraftCube locket (Wallace. 2006), that do not necessarily connect to other devices and expand on the traditional role of mementos. But it would also be interesting to see what interaction scenarios could immerge in the context when a piece of sentimental jewelry on a wearer becomes computationally connected to the giver, transforming its "analogue" 1-way interaction into synchronous or asynchronous 2-way interaction.

\subsection{User Groups \& Interaction Scenarios}

Our study clearly indicates that women are both the most likely receivers and wearers of sentimental jewelry, suggesting that there is an opportunity to engage with currently understudied users of wearables. It was valuable to confirm that romantic partners are still predominant users of sentimental jewelry, which aligns with many use-cases in CMC between intimate relationships (Hassenzahl et al., 2012) and that sentimental jewelry is largely used for communication of affection. However, we see that these artefacts are also widely used to mediate emotional connection with friends and close family members for wishes of good luck and motivation as important factors that create a sense of connectedness between people. This aligns with theories in relationship psychology that talk about importance of every-day maintenance and support for thriving through relationships (Feeney \& Collins, 2014). In this view, the research on social wearables could be extended from its predominant use in LDR into areas such as wellbeing, health and personal security, which could benefit from utilizing connectedness to loved-ones in their design. And recent female safety and wellbeing devices like artemisfashion.com and bellabeat.com point at the fact that this is already happening.

The wider user groups might be particularly relevant to aid in increasing the sense of connectedness with aging population and encourage their independence and wellbeing. It might be interesting to investigate coupling jewelry form-factor with motivation or good luck elements and introducing an element of ambiguity and enchantment (Mccarthy et al., 2005) in the design of social wearables could transcending the differences in the adoption of technology between generations. As motivators, these groups might also play a role in the areas that look at health, wellbeing and fitness. And it is also worth to consider that based on current use of sentimental jewelry, these 2-way interactions through social wearables will likely involve a wearer of smart jewelry (female) and loved-one (male or female) who would use a non-wearable device, such as smart phone.

\section{CONCLUSION}

Keeping in touch and maintaining relationships with loved ones is an essential component of wellbeing. For millennia, people used sentimental jewelry to create a meaningful sense of connectedness. The field of social wearables echoes this practice and offers novel interactions and possibilities, many of which are yet to be studied. But the digital artefacts currently created in this field often have a gadgetlike form-factor and are easily discarded. In this paper, we looked at what lessons we can derive from modern use of "analogue" sentimental jewelry, and how these could be applied to the design and research practice on social wearables. We uncover that in contrast to current social wearables, analogue artefacts are rarely paired and intended to be worn predominantly by female partners, friends and family members. Many explicitly convey affection, good luck or motivation, but are seldom personalized, and allow users to infuse their own meaning into artefacts. Based on the results, we propose possible new design directions and interaction scenarios for future research that can lead to the creation of meaningful and long-lasting social wearables that mediate relationships between loved-ones. 


\section{ACKNOWLEDGEMENTS}

This research is funded by Queen Mary University of London, Media Arts Technology group.

\section{REFERENCES}

Petra Ahde-Deal. 2013. Women and Jewelry: The Social Approach to Wearing and Possessing Jewelry. Ph.D. Aalto University, Helsinki Finland.

Shirley Bury. 1986. An Introduction to Sentimental Jewellery. V\&A. Stationery Office Books

Brooke C Feeney and Nancy L Collins. 2014. A New Look at Social Support: A Theoretical Perspective on Thriving Through Relationships. Personality and Social Psychology Review. doi:10.1177/1088868314544222.

Marc Hassenzahl, Stephanie Heidecker, Kai Eckoldt, Sarah Diefenbach, and Uwe Hillmann. 2012. All You Need Is Love: Current Strategies of Mediating Intimate Relationships through Technology. ACM Transactions on ComputerHuman Interaction, Vol. 19, No. 4, Article 3019 (4): 30. doi:10.1145/2395131.2395137.

Sarah Kettley. 2007. Crafting the Wearable Computer: Design Process and User Experience. Ph.D. Centre for Interaction Design Napier University, Edinburgh UK.

Ruth Kikin-Gil. 2005. BuddyBeads : TechnoJewelry for Non Verbal Communication within Groups of Teenage Girls. In MobileHCl' 05 Proceedings of the International Conference on Human-Computer Interaction with Mobile Devices and Services Adjunct. - 10 (2-3).

Kahyun Sophie Kim, Anna-Marie Mansour, Jay W. Lundell. 2016. Lessons learned from designing a displayless consumer wearable tech. September In MobileHCl '16 Proceedings of the International Conference on Human-Computer Interaction with Mobile Devices and Services Adjunct.
John Mccarthy, Peter Wright, Jayne Wallace, and Andy Dearden. 2005. The Experience of Enchantment in Human - Computer Interaction. Personal and Ubiquitous Computing 10: 369-78. doi:10.1007/s00779-005-0055-2.

Geoffrey C. Munn. 1993. The Triumph of Love. Thames \& Hudson Ltd

Mah Rana. Ongoing. Meanings and Attachments. Retrieved April 11, 2018 from www.meaningsandattachments.com

Diana Scarisbrick and James Fenton. 2013. Rings: Jewelry of Power, Love and Loyalty. Thames and Hudson Ltd

Sabine Seymour. 2008. Fashionable Technology: The Intersection of Design, Fashion, Science, and Technology. Walter de Gruyter \& Co.

Yulia Silina and Hamed Haddadi. 2015. 'New Directions in Jewelry': A Close Look at Emerging Trends \& Developments in Jewelry-like Wearable Devices. In ISWC '15 Proceedings.

Thad Starner. 2001. The challenges of wearable computing: Part 2. IEEE Micro, 21(4), 54-67.

Hugh Tait and Carol Andrews. 2007. 7000 Years of Jewellery. British Museum Press; 2 edition

Maarten Versteeg, Elise van den Hoven, \& Caroline Hummels. 2016. Interactive Jewellery: a design exploration. In TEl ' 16 Proceedings.

Jane Wallace. 2006. CraftCube Locket. Retrieved April 11, 2018 from www.digitaljewellery.com/jaynewallace/CraftCube _Locket.html

Jane Wallace. 2007. Emotionally Charged: A Practice-Centred Enquiry of Digital Jewellery and Personal Emotional Significance. Ph.D Dissertation. Sheffield Hallam University, Sheffield UK 